\title{
Infinity, Technology, Degeneracy: A Note on Werkhoven's Dispositional Theory of Health
}

\author{
Shane N. Glackin
}

\begin{abstract}
Sander Werkhoven's ‘A Dispositional Theory of Health' is an important and original contribution to debates about the disease concept, which persuasively demonstrates that dispositions must play some role in a full account of what it is to be healthy or ill. Unfortunately, as a theory, it cannot as it stands be correct. I first demonstrate what appears to be a significant, and possibly fatal, flaw; the proliferation of dispositions which Werkhoven's theory requires makes impossible, at least in the absence of significant further metaphysical work, the comparative numerical judgements on which its account of health and illness are based. I then demonstrate two further problems, concerning the exclusion of 'technological' dispositions from those under consideration, and a large class of compensatory biological functions which Werkhoven's theory seems to have overlooked.
\end{abstract}

\section{Infinite Variety \\ II. L'Homme Machine}

III. TIMTOWTDI

IV. Conclusion

Sander Werkhoven's 'A Dispositional Theory of Health' ([2018]) is an important and original contribution to debates about the disease concept. It accords impressively with intuitions about the disease-status of particular conditions, and thereby persuasively demonstrates that dispositions must play some role in a full account of what it is to be healthy, or ill. ${ }^{1}$ Unfortunately, as a theory, it cannot as it stands be correct.

\footnotetext{
${ }^{1}$ For the purposes of this discussion note I shall use 'illness' and 'disease' interchangeably; though the practice is disputed in the literature, nothing important hangs on it in this case.
} 
There is one primary reason for this, which I shall explain in Section I; the proliferation of fine-grained dispositions which Werkhoven's theory requires to explain comparative health makes impossible the comparative numerical judgements on which its account of health and illness are based, at least until a significant metaphysical problem is resolved. There are two more problems for the theory, albeit less significant ones, which I outline in Sections II and III: the first, shared by some rival theories, concerns its reliance on kinds and reference classes, and in particular a somewhat arbitrary exclusion of 'technological' dispositions from those under consideration; the second concerns a large class of compensatory biological functions, which seem to be effectively overlooked by Werkhoven's theory.

\section{Infinite Variety}

Health and disease, according to Werkhoven, are a matter of one's capacities; or, to use the philosophers' technical jargon, one's 'dispositions'. It is in the loss of these that illness consists, ${ }^{2}$ and in their full or partial restoration that being healed or cured does. Health, he says, is therefore 'a certain measure of what a living organism can and cannot do', relative to the most we can expect an organism of its sort to be able to do (Werkhoven [2018], p. 7). This is certainly an intuitively attractive view.

Problems emerge as Werkhoven proceeds to elaborate the attractive, but somewhat vague, intuition here more formally. He provides a linked set of definitions, the first of which is for the non-comparative notion of 'health';

(2) Health: Health is the ratio of a living organism's dispositional set compared to the maximum dispositional set belonging to its reference class. ([2018], p. 8)

The second is for a derived, comparative concept of 'healthier than';

\footnotetext{
2 'A shoulder lesion prevents one from lifting one's arm; intermittent claudication reduces the ability to walk; Broca aphasia constrains the capacity for speech, anxiety disorders limit people in specific actions (like appearing in public, or entering airplanes); depressions inhibit people more generally in activities of daily living (like getting dressed, preparing food, going to work, and so on); and so on'. (Werkhoven [2018], p. 3).
} 
(3) Healthier Than: $X$ is healthier than $Y$ if and only if $X^{\prime}$ s ratio ( $X^{\prime}$ s actual dispositional set compared to $X$ 's maximum possible dispositional set) is higher than $Y^{\prime}$ s ratio ( $Y$ 's actual dispositional set compared to $Y^{\prime}$ 's maximum possible dispositional set). ([2018], p. 8)

The third, finally, is for a 'more loosely comparative' concept involving 'a comparison not to one concrete individual, but to a wider comparison class';

(4) Healthy: $X$ is healthy ${ }^{3}$ if and only if $X$ 's ratio ( $X$ 's actual dispositional set compared to $X$ 's maximum possible dispositional set) is larger than the averaged ratio of a sufficiently large number of members of the relevant comparison class. ([2018], p. 9)

Already, with the talk of ratios in each of these definitions, a potentially fatal problem has emerged. To see why, consider the capacity, or disposition, to walk. To support the sort of comparative judgement Werkhoven wants to account for, this needs to be understood as not one, but many very fine-grained dispositions; somebody who can sustain a walking speed of $4.5 \mathrm{~km} / \mathrm{h}$ is less healthy than someone with the disposition to walk at $5 \mathrm{~km} / \mathrm{h}$, but healthier than someone who is disposed only to walk at $4 \mathrm{~km} / \mathrm{h}$. All three are healthier than someone who lacks the disposition to walk altogether. But someone who can walk at $4.5 \mathrm{~km} / \mathrm{h}$ for 45 minutes is healthier than someone who can only do so for half an hour, and less healthy than someone who can do so for 50, or 55 minutes, or an hour or longer. A competitive race-walker might regard himself as in poor health compared to last season if he can no longer walk at more than $12.5 \mathrm{~km} / \mathrm{h}$ for four hours at a stretch. People who can walk for an hour on very undulating ground are healthier than people who can do so only on mildly undulating ground, who are healthier again than those who can do so only on the flat. One can easily come up with many more such differentiating factors: being able to walk at a given pace for a given time in any pair of shoes, or only in some types; being able to walk with some quantities and types of food intake; being able to walk while talking, or without talking, or only talking a little, and so on. 4

\footnotetext{
3 A linked definition (5) gives 'unhealthy' mutatis mutandis (Werkhoven [2018], p. 9).

${ }_{4}$ Some important capacities seem to be environment-relative, moreover; the ability to walk to the nearest shop, post office, bus-stop etc. may be important health-related dispositions, losing any of
} 
Indexing dispositions to any such differentiating factor will clearly generate a host of finegrained distinctions. So already we can see how making standard comparative judgements of health and illness requires that we regard walking, or any similarly health-related ability, not as a single coarse-grained capacity, but rather as a myriad of much more fine-grained capacities. And this makes the work to which Werkhoven wishes to put ratios look impossible. The set of dispositions which any organism possesses is evidently infinite; it has a cardinality of $\aleph_{0}$. Indeed, Werkhoven admits as much when he describes a frog's reproductive dispositions as falling 'on a rational number between zero and one, depending on how much spawn it is able to produce' ([2018], p. 7, fn. 15). Even if he is correct that illness is a matter of subtracting dispositions from the total, therefore, the subtraction does not reduce the total quantity. The maximum possible dispositional set for the organism's reference class has the same cardinality, so the ratio of the two is $1: 1$ in every case; every living organism is therefore maximally healthy. The ratio for every other living organism is also 1:1, so every pair of organisms is equally healthy. The averaged ratio for any comparison class will likewise be 1:1, so no organism will in the 'more loosely comparative' sense ever be either healthy or unhealthy. In short, while Werkhoven's intuition about pathology eliminating dispositional capacities is a plausible and attractive one, the attempt to precisify it mathematically requires invoking a multitude of fine-grained dispositional capacities which ultimately render it selfdefeating.

Obviously, dispositionalists will wish to resist this kind of proliferation. The disposition of fragility, for instance, should not entail the separate dispositions to break into 2, 10, or 100 pieces. The usual remedy for this is to talk of dispositions 'coming in degree'; fragility, like the disposition to walk, is unitary, but it is possessed in different intensities and strengths by different individuals. ${ }^{5}$ However, this response does not seem to be available to Werkhoven. To explain why the different walkers described above differ in their states of health, his numerical method requires them to differ in the number of dispositions they possess. But once we allow that the number of dispositions in question is therefore infinite, it is just in this respect that they cannot differ.

which will significantly impact on one's quality of life, even though the distances in question will be different for different individuals. These are potentially less problematic for Werkhoven, as the dispositional theory of health may be construed as restricting the properties under consideration to the intrinsic properties of the organism. However, several recent papers (Davies 2016; Glackin 2017; Roberts et al. 2019) have argued that health and disease are at least sometimes externallyconstituted. If these arguments are correct, then a dispositionalism which excludes environmentrelative properties would face a further problem.

5 I am indebted to Andrea Raimondi for illuminating discussion of this point. 
In his Section 3.3 ([2018], pp. 12-4), responding to a different concern, Werkhoven gestures towards what looks like a potential solution. '(N)ot every disposition is equally relevant when it comes to health', he writes; '( $\mathrm{t}$ )he disposition to wiggle one's little toe independently of other toes, for instance, is much less important to human health than the disposition to walk, to communicate, or to form true beliefs about the world' (p. 12). And losing the disposition to wiggle one's little toe is a much less substantial loss of health than losing the ability to walk or speak. This apparently qualitative difference can be explained, Werkhoven argues, by recognizing the hierarchical dependencies among our dispositions. 'The disposition to walk', for instance, 'is required for the disposition to run up and down staircases, to move an object from one place to another, to engage in various social activities, and so on, as well as the whole range of dispositions made possible by these more specific dispositions' (p. 13), whereas very little else hangs on the disposition to wiggle one's little toe. Could Werkhoven, then, salvage his theory by arguing that differences in strength and intensity in the unitary disposition to walk lead to differences in the number of subsidiary dispositions thereby enabled?

Alas, this would be a hollow victory, since the same problem would merely recur one stage later. The number of things that walking enables us to do is, again, infinite. So subtracting any subset of such subsidiary dispositions from an organism's total set again leaves it with the same overall quantity, and the ratios remain unchanged.

Perhaps this line of criticism is ultimately unfair, however. Werkhoven admits in his conclusion that more 'must ... be said about the individuation of dispositions, which will require further in-depth metaphysical reflection' (p. 23). But this may look like a problem for metaphysicians, and not philosophers of medicine, to solve; and so it would be unfair to criticize a philosopher of medicine's outline for a theory of health for not yet being able to make use of suitable theory of property-individuation, just as it would be unfair to criticize utilitarians for not yet having a clear view of how to quantify utility, or well-being, or whatever is to be aggregated. ${ }^{6}$

I'm not entirely persuaded by this defence, not least because I think this plausibly is a legitimate criticism of consequentialist theories of morality; one philosopher's ponens is another's tollens. Moreover, I think it's tricky even to say with confidence what exactly Werkhoven's theory of health ultimately involves unless we do have a clear picture of how the metaphysics are supposed to work here. And Werkhoven's discussion of the spawning frog, whose set of possible reproductive dispositions has the cardinality of the rational numbers, looks like the proverbial 'smoking text' to me. But there's a tu quoque available to my critic

\footnotetext{
${ }^{6}$ I thank an anonymous referee for raising this point, as well as for suggesting the ensuing reformulation of my argument.
} 
here as well; perhaps Werkhoven has failed to show something his theory requires, which is that dispositions can be individuated in the appropriate fashion without explosion, but I have equally failed to provide an explicit argument for my own contrary supposition that every object (or at least, every organism) will turn out to have infinite dispositions. Neither of us, then, has supplied the necessary metaphysical workings.

A reader finding this line of argument persuasive can nevertheless understand my argument here as a conditional challenge; if, or as long as, we don't have a satisfactory account of how to individuate dispositional properties, then the multitude of fine-grained distinctions in capacity which we observe will yield an infinity of dispositional properties, rendering all individuals equally and optimally healthy by Werkhoven's criterion. So perhaps Werkhoven's theory can be rescued simply by supplying more metaphysical clarity or precision on this question; but until that clarity or precision is forthcoming, it is in trouble.

In the continuing absence of such elaboration, at any rate, there seems to be no rescuing Werkhoven's explicitly numerical dispositionalist account of health from this problem. It is intuitively correct that illness-perhaps necessarily and constitutively-involves an overall reduction in one's capacities compared to a state of health. But the arithmetic of infinite quantities is very far from intuitive. What Werkhoven-or any dispositionalist-needs is a criterion of relevance; a way of specifying the finite set of dispositions with which judgements of health and illness are concerned. But such a criterion is, approximately at least, what all the other accounts of health and illness that his is supposed to supersede ${ }^{7}$ have been trying to provide. And what the preceding argument has shown is that, even with a satisfactory criterion of relevance in place, the problem of fine-grainedness means that while no such finite set seems to be available, so a quantitative approach to definitions of health and illness cannot succeed.

\section{L'Homme Machine}

A second problem for Werkhoven's account-not, I think, as potentially damaging as the first, but certainly enough to weigh against it in the cost-benefit analysis of competing theories-is its reliance on biological kinds and reference classes. The basic ratio defined in (2), on which the other definitions depend, follows Christopher Boorse's 'biostatistical theory' (Boorse [1975], [1977]) of health and disease by invoking the organism's 'reference class', defined as an age-group of a sex of a species ([2018], p. 10).

\footnotetext{
${ }_{7}$ A helpful, non-exhaustive list of these is provided at the start of (Werkhoven [2018])'s p. 2.
} 
Boorse's use of reference classes has, however, been extensively criticized in the literature. ${ }^{8}$ Werkhoven does note one particularly damaging criticism ([2018], p. 10, fn. 16), due to Elselijn Kingma ([2007]); the only non-arbitrary basis for limiting the specification of the reference class to these three categories-and not, for instance, judging someone's liver function to be healthy by comparison to other alcoholic males aged 40-50-must be that being a woman, or a child, is not a disease-state, whereas being an alcoholic is. But Boorse's theory, like Werkhoven's, is supposed to tell us which conditions are diseases and which are healthy; it is viciously circular for the definitions of disease and health to presume prior knowledge of that distinction. Werkhoven does gesture towards an answer to the charge that the reference classes thereby introduce implicit social biases and values, but he does not offer any defence against the charge of circularity, nor against the various other objections that have been made to Boorse's account; for the time being, at least, these must count against acceptance of the dispositional theory too.

As well as inheriting these problems, Werkhoven's theory raises another of its own in the way that the organism's 'biological kind' is specified. This is an unavoidable consequence of considering dispositions. As Michael Tooley ([1972]) has noted in another context, just about any biological organism has the disposition, or 'potentiality'-given the right environmental conditions-to realize just about any biological state; a kitten injected with the right-currently unknown, science-fictional-serums, and provided with suitable nutrition and developmental environment, would achieve the same mental sophistication as an adult human. So to avoid comparing the dispositional sets of actual living persons with the infinite range of dispositions speculatively achievable in principle with the aid of esoteric future science, and thereby rendering his measure of health and illness practically meaningless, Werkhoven must delimit the maximum dispositional set of the reference class to those dispositions currently attainable by members of the class without the assistance of technology; '(d)ispositions gained through various forms of enhancements-drugs, prostheses, exoskeletons, and so on-are not to be included: the relevant maximum is that belonging to the species, not that of a technologyenhanced species' ([2018], p. 7).

One problem here involves the idea-which I think survives Werkhoven's denial that his view requires essentialism about species-that there is some determinate way for the members of a species or reference class to be that is appropriate to use as a measure for all of them; this is again not unique to Werkhoven's theory, and has been previously criticized in the literature (see, for example, Lewens [2010]; Glackin [2016]). Another potential issue is that dispositions seem to exist whether or not the relevant environmental triggers do-the brittle window-pane

\footnotetext{
8 For useful recent overviews see (Griffiths and Matthewson [2018], s. 4) and (Murphy [2015]).
} 
has the disposition to be broken by stray footballs even in possible worlds where footballs have not been invented-meaning that the set of dispositions an organism possesses is plausibly unaffected by the state of the technology which might cause them to be realized. But a more novel problem concerns the exclusion of 'technology-enhanced species' as a matter for concern.

The problem is this; humans are already a technology-enhanced species, and the intuitions about our health that Werkhoven wishes to explain are crucially dependent on those technologies. (see, for example, Haraway [1991]; Latour [1993]). ${ }^{9}$ The dispositions to read and write', according to Werkhoven, '[...] are basic dispositions, meaning that on my account illiteracy would make for a substantial health-deficit' ([2018], p. 23). But if reading and writing are not technologies, ${ }^{10}$ nothing is! A modern emotional life, moreover, shaped as it is by art and literature, and necessary for successful participation in a modern cultural environment, is no less a product of those technologies, while our epistemic and cognitive functions likewise increasingly depend on the information made available by modern communications technology. Lactose-tolerance is an adaptation to the technologies of dairy-farming and the domestication of cattle; while our microbiota are shaped profoundly by newer technologies such as pasteurization. Transportation technologies are essential to human gene flows-not for nothing did historian Eric Hobsbawm describe the bicycle as 'the greatest single device for achieving ... the full realisation of being human invented since Gutenberg' ([2002], p. 89)while mobility in modern urban environments even often requires any disposition to walk less than those relevant to driving. A credible account of my own health-related dispositions surely takes into proper account my use of orthotic insoles and reading-glasses. And it is notoriously difficult, in any case, to draw a principled line between the technological 'enhancements'

\footnotetext{
9 As, arguably, are those species we have domesticated, plus several others who have developed a capacity for tool use.

${ }^{10}$ To be clear; I mean here that the system of conventions and practices we use for visually (or tactilely) encoding and decoding semantic information are a technology, not merely that the media we use for doing so-such as pens and paper-are. I thank an anonymous referee for pointing out the ambiguity here. A reader might still suppose that a relevant distinction is being obscured here, between the cognitive capacities to read and write on the one hand, which are possessed by individuals, and the broader socio-technical institution of writing. I'm unpersuaded that such a distinction can be drawn, but in either case the problem for Werkhoven is the same; he is caught between the Scylla of acknowledging that technically-augmented environments make it the case that some individuals have greater capacities than others, and the Charybdis of maintaining that those with lesser capacities are rightly regarded as pathological. I thank the same referee for suggesting both this objection and its solution.
} 
Werkhoven wishes to exclude, and the medical interventions he already recognizes as properly restoring the dispositions we lose to illness (see, for example, Resnik [2000]).

Again, some such problem seems endemic to Werkhoven's approach, and so unavoidable. He must limit the dispositions under consideration to those currently achievable, on pain of proliferation. But doing so will always run into conflict with the fact that biological species are dynamic rather than static entities, eternally subject to change and development. Biological nature, in short, is as Hopkins wrote 'a Heraclitean fire' (1888; see the essays in Nicholson and Dupré 2018); so a worthwhile account of what it is that medical classifications aim to capture cannot work by freezing them in time.

\section{TIMTOWTDI'11}

There is a final problem with Werkhoven's account. Again, it is not fatal, and it weighs less heavily in the balance of theoretical costs and benefits than the last considered; but it weighs all the same. It is a problem that Werkhoven does recognize, and respond quite persuasively to. However, I will show, the true scale of this problem is much greater than he has supposed; and this will in turn make his defensive strategy rather less attractive to many readers.

One objection Werkhoven imagines to his holistic view of health is 'that pathology-induced reductions of what one can do can be compensated for by developing compensatory mechanisms, to the point that one's overall dispositional is returned to its original state, despite the local defect not having been remedied' ([2018], p. 21). He gives the example of a person who can no longer grip with their left hand, but who compensates for it by developing a series of alternative manoeuvres and tricks with their 'right hand, elbow, feet, mouth, and so on' to the point that they are no longer impaired with regard to their functional capacities 'as a whole' ([2018], p. 21). He bites the bullet on this question, albeit with reservations; full restoration of dispositions would count as full restoration as health, but

(i)t is questionable [...] whether such extensive and complete compensation can ever become reality, or whether this is indeed only a theoretical possibility. No matter how skilful and deft one becomes, certain activities will be precluded when one hand has lost its gripping function: carrying heavy items in both hands, cutting a pumpkin on a countertop, playing the oboe, and so on-enough activities are excluded to reduce the ratio of actual to maximum possible dispositional sets to support the verdict of a pathology. ([2018], p. 21)

${ }_{11}^{11}$ 'There is more than one way to do it'; a motto in PERL programming. 
But functional compensation is a far more ubiquitous biological phenomenon than this example suggests. One comparatively obvious, but comparatively rare, manifestation of this is in biological 'redundancy'. Humans have two kidneys. We can perform perfectly well with one, which is why some heroic individuals are able to donate them to others during life; so losing a kidney involves no clear loss of dispositions. ${ }^{12}$ On the other hand, losing a kidney seems clearly to represent an impairment of health, which is precisely why we regard those who do so voluntarily as heroic. Indeed, it would appear absurd to hold that the very same biological condition was an illness only when present in one's second, or one's sole (post-donation, or post previous-illness) kidney, but not when present only in the first. Probably not every reader will find this as counterintuitive as I do; but for those who do, this is a problematic implication of Werkhoven's theory.

Far more common, but little-known, than redundancies of this sort are cases of biological 'degeneracy'. ${ }^{13}$ Degeneracy, in its technical biological sense, refers to the ability 'of elements that are structurally different to perform the same function or yield the same output' (Edelmann and Gally [2001], p. 13763), or 'of different structures to be conditionally interchangeable in their contribution to system functions' (Mason [2014], p. 12), and is a key element of the functional plasticity of organisms. It differs from redundancy in that functional structures are not duplicated, but rather the same functional effect is achieved by different structures. This is an extremely widespread phenomenon, and a foundational concept in systems biology and related fields. And it poses a problem for Werkhoven. Whether or not rival views 'can claim an advantage' regarding cases of functional compensation, he writes, 'depends on the genuine possibility of such a scenario' (Werkhoven [2018], p. 21), and he professes himself 'sceptical' (p. 22) on the point. Such potentially advantage-shifting cases turn out not to be merely possible, however, but virtually ubiquitous. A great many of any organism's parts overlap in their functional disposition, and so can compensate at a holistic level for perturbations to each other. It remains open to Werkhoven-and any reader-to bite the bullet here, as he did in the 'weakened left hand' case, and insist that cases where the overall action-potential is holistically preserved are thereby not cases of illness. But given the evident ubiquity of such cases, the total amount of dietary lead involved is clearly much greater than Werkhoven initially envisaged; what appears palatable in isolated, contrived cases may

\footnotetext{
12 If anything, losing a kidney provides me with rather more dispositions than I previously possessed; the disposition to become seriously ill if the other one fails, for instance. But there certainly doesn't seem to be the sort of dispositional loss that Werkhoven suggests is likely to occur in real-world cases of functional compensation.

13 I am grateful to Çağlar Karaca for this observation.
} 
look very different in bulk. So this is not a 'knock-down' argument, and the determined dispositionalist will not be dissuaded by the spectre of degeneracy. For many readers not already convinced by dispositionalism, however, it will provide a further reason to resist Werkhoven's conclusions.

\section{Conclusion}

Though I have been critical of it here, Werkhoven's paper represents an important contribution to, and significantly advances, philosophical debates about the disease concept. Those debates have long been dominated by the Biostatistical and Harmful Dysfunction theories, and have by now settled into the stasis and endless proliferation of examples and counterexamples that characterizes 'mature' philosophical disputes. New perspectives and approaches are desperately needed to break the stalemate, and while I find the details of Werkhoven's proposal to be unworkable, its focus on human capacities breaks new ground in both fitting with, and explaining in plausible terms, our intuitions about health and disease conditions. Dispositions, I am now persuaded, must play some role in any satisfactory account of the disease concept; they just can't do it in this way.

\section{Acknowledgements}

My thanks to Andrea Raimondi for helpful discussion of the dispositions literature, and comments on a draft of this paper, as well as to the journal's editors and anonymous reviewers; all significantly improved the finished article.

Shane N. Glackin EGENIS/Dept. of Sociology, Philosophy, and Anthropology University of Exeter, United Kingdom s.n.glackin@exeter.ac.uk 


\section{References}

Boorse, Christopher [1975]: 'On the Distinction between Disease and Illness', Philosophy and Public Affairs, 5, pp. 49-68.

-- [1977]: 'Health As a Theoretical Concept', Philosophy of Science, 44, pp. 542-73.

Davies, William [2016]: 'Externalist Psychiatry', Analysis, 76, pp. 290-6.

Edelman, Gerald M. and Gally, Joseph A. [2001]: 'Degeneracy and Complexity in Biological Systems', Proceedings of the National Academy of Sciences of the United States of America, 98(24), pp. 13763-8.

Glackin, Shane N. [2016]: 'Three Aristotelian Accounts of Disease and Disability', The Journal of Applied Philosophy, 33(3), pp. 311-26.

-- [2017]: 'Individualism and the Medical: What about Somatic Externalism?', Analysis, 77, pp. 287-93.

Griffiths, Paul E. and Matthewson, John [2018]: 'Evolution, Dysfunction, and Disease: A Reappraisal', British Journal for the Philosophy of Science, 69, pp. 301-27.

Haraway, Donna J. [1991]: Simians, Cyborgs, and Women: The Reinvention of Nature. New York: Routledge.

Hobsbawm, Eric [2002]: Interesting Times: A Twentieth-Century Life, London: Allen Lane.

Hopkins, Gerard Manley [1888]: 'That Nature is a Heraclitean Fire and of the Comfort of the Resurrection', in The Major Works (2009), trans Catherine Phillips. Oxford: Oxford World's Classics.

Kingma, Elselijn [2007]: 'What Is It to Be Healthy?', Analysis, 67, pp. 128-33.

Latour, Bruno [1993]: We Have Never Been Modern, trans Catherine Porter. Harvard: Harvard University Press.

Lewens, Tim [2010]: 'Foot Note', Analysis, 7o(3), pp. 468-73.

Mason, Paul H. [2015]: 'Degeneracy: Demystifying and Destigmatizing a Core Concept in Systems Biology', Complexity 2o(3), pp. 12-21.

Murphy, Dominic [2015]: 'Concepts of Disease and Health', in E.N. Zalta (ed.), Stanford Encyclopedia of Philosophy, available at $<$ https://plato.stanford.edu/archives/spr2015/entries/health-disease/>. 
Nicholson, Daniel J. and Dupré, John (ed.s) [2018]: Everything Flows: Towards a Processual Philosophy of Biology. Oxford: Oxford University Press.

Resnik, David B. [2000]: 'The Moral Significance of the Therapy-Enhancement Distinction in Human Genetics', Cambridge Quarterly of Healthcare Ethics, 9(3), pp. 365-77.

Roberts, Tom, Krueger, Joel, and Glackin, Shane N. [2019]: 'Psychiatry Beyond the Brain: Externalism, Mental Health, and Autistic Spectrum Disorder', Philosophy, Psychiatry, \& Psychology (forthcoming, online first).

Tooley, Michael [1972]: ‘Abortion and Infanticide’, Philosophy \& Public Affairs, 2(1), pp. 3765.

Werkhoven, Sander [2018]: 'A Dispositional Theory of Health', British Journal for the Philosophy of Science, Online First. 